\title{
Studies on Heterosis for Yield and its Contributing Traits in Sponge Gourd (Luffa cylindrica Roem.)
}

\author{
Vijay Bahadur Singh Chauhan ${ }^{1,2^{*}}$, Dinesh Kumar $\operatorname{Singh}^{1}$ and \\ Harshawardhan Choudhary ${ }^{1,3}$
}

${ }^{1}$ Department of Vegetable Science, Govind Ballabh Pant University of Agriculture and Technology, Pantnagar-263145, U.S. Nagar, Uttarakhand, India

${ }^{2}$ ICAR-Central Tuber Crops Research Institute, Regional Centre, Bhubaneswar-751019, Odisha, India

${ }^{3}$ ICAR-Indian Agricultural Research Institute, New Delhi-110012, India

*Corresponding author

A B S T R A C T

\section{Ke y w ords \\ Sponge gourd, Luffa \\ cylindrical, Half diallel, \\ Heterosis, Hybrid, Yield \\ traits \\ Article Info \\ Accepted: \\ 04 November 2018 \\ Available Online: \\ 10 December 2018}

The present study was carried out on heterosis for fruit yield and yield related traits in sponge gourd. Fifteen hybrids of sponge gourd were developed using six parents in half diallel fashion were studied for estimation of heterosis for 10 yield contributing traits. Hybrids PSG-82 × PSG-07-04, PSG-07-04 × PSG-161 and PSG-07-04 × PSG-199 were found most promising for earliness and yield. These hybrids exhibited heterosis to the tune of 59.36, 48.45 and 43.85 per cent over their better parents and 81.58, 69.16 and 63.91per cent, respectively over the check cultivar for fruit yield per hectare. The cross combination PSG-82 × PSG-07-04 recorded highest significant heterobeltiosis for yield and its contributing traits and it was identified for evaluation in multi-location trial and commercial exploitation.

\section{Introduction}

Smooth gourd (Luffa cylindrica Roem.) is one of the important member of the family Cucurbitaceae and originated in subtropical Asian Region particularly India (Kalloo, 1993). This is an annual plant, which produces fruits containing a fibrous vascular system. It is commonly grown for its tender fruits as well as for sponge which is used for scrubbing purposes. The dried fruits are used as a bath sponge, increase blood circulation and credited as a relief for rheumatic and arthritic suffers. Tender fruits are rich in vitamin A, vitamin $\mathrm{C}$ and iron (Yawalker, 2004). It is highly cross pollinated due to monoecious nature. Various breeding techniques have been advocated considering the breeding behaviour of crop.

Out of these hybrid breeding is prominent and used in the improvement of vegetable crops, especially in out breeding species (Kalloo et al., 2000). So a speedy improvement can be brought by exploiting heterosis for various yield contributing traits as well as earliness. 
However, the information related to hybrid development in sponge gourd is very limited in India. Keeping above facts in consideration, the present investigation was carried out to estimate the extent of heterosis for different yield attributing traits.

\section{Materials and Methods}

Six genetically diverse parental lines of sponge gourd were used to develop $15 \mathrm{~F}_{1} \mathrm{~s}$ following a half diallel mating system, excluding reciprocals during Kharif season. The parental lines used were PSG-93 $\left(\mathrm{P}_{1}\right)$, PSG-82 ( $\left.\mathrm{P}_{2}\right)$, PSG-07-04 ( $\left.\mathrm{P}_{3}\right)$, PSG-115 $\left(\mathrm{P}_{4}\right)$, PSG-199 ( $\left.\mathrm{P}_{5}\right)$ and PSG-161 $\left(\mathrm{P}_{6}\right)$. The $15 \mathrm{~F}_{1} \mathrm{~S}$ along with their six parents were evaluated during summer season 2008 at the Vegetable Research Centre of the G.B. Pant University of Agriculture and Technology, Pantnagar, U.S. Nagar, Uttarakhand, India.

The experiment was laid out in a randomized block design (RBD) with three replications. Each genotype was grown in a single row of 8 $\mathrm{m}$ length with a spacing of $3 \times 1 \mathrm{~m}$. There were eight hills per entry with one plant at each hill. Observations for ten horticultural traits were recorded on five randomly selected plants.

The traits under study were days to anthesis of first male flower, node number of first male flower, days to anthesis of first female flower, node number of first female flower, fruit length, fruit weight, number of fruits per plant, number of primary branches per plant, main vine length and fruit yield per hectare. The mean data obtained for quantitative traits on per plant basis were analysed statistically for heterosis as suggested by Fonseca and Patterson (1968).

\section{Results and Discussion}

There were significant differences among the parental lines with respect to different characters studied including total yield per hectare. The mean performance of six parental lines along with $15 \mathrm{~F}_{1}$ hybrids is given in Table 1 . The mean values of parents for days to first male flower anthesis ranged from $51.67\left(\mathrm{P}_{4}\right)$ to 67.67 days $\left(\mathrm{P}_{1}\right)$ and among crosses it ranged from $49.33\left(\mathrm{P}_{1} \times \mathrm{P}_{3}\right)$ to 69.67 days $\left(\mathrm{P}_{2} \times \mathrm{P}_{6}\right)$. Out of $15 \mathrm{~F}_{1}$ hybrids, a total of 5 and 10 crosses showed negative heterosis over better parent and mid parent, respectively. The hybrid $\mathrm{P}_{1} \times \mathrm{P}_{2}$ exhibited maximum heterosis of $-18.81 \%$ and $-19.01 \%$ over better parent and mid parent, respectively. With respect to node number of first male flower, parental mean ranged from $4.33\left(\mathrm{P}_{4}\right)$ to $10.67\left(\mathrm{P}_{2}\right)$. Among the crosses it ranged from $3.67\left(\mathrm{P}_{1} \times \mathrm{P}_{3}\right)$ to $12.67\left(\mathrm{P}_{5} \times \mathrm{P}_{6}\right)$ node number.

The extent of heterosis varied from -19.23 to $53.84 \%$ over better parent and -12.00 to $54.71 \%$ over mid parent. Six $F_{1}$ hybrids showed heterosis over better parent, out of which $\mathrm{F}_{1}$ hybrid $\mathrm{P}_{1} \times \mathrm{P}_{5}$ showed maximum heterosis over better parent $(-53.84 \%)$ and mid parent (-54.71\%) while $\mathrm{F}_{1}$ hybrids $\mathrm{P}_{2} \times \mathrm{P}_{3}, \mathrm{P}_{3}$ $\times \mathrm{P}_{6}$ and $\mathrm{P}_{3} \times \mathrm{P}_{4}$ showed maximum heterosis over standard parent $(23.17 \%)$. The mean values of parents for days to first female flower anthesis ranged from $52.67\left(\mathrm{P}_{3}\right)$ to 77.33 days $\left(\mathrm{P}_{1}\right)$. Out of $15 \mathrm{~F}_{1}$ hybrids, 5, crosses showed negative heterosis over better parent, 2 over standard parent and 9 over mid parent. The $\mathrm{F}_{1}$ hybrid $\mathrm{P}_{1} \times \mathrm{P}_{2}$ showed maximum heterosis over better parent ($18.55 \%)$ and mid parent $(-20.53 \%)$ while $F_{1}$ hybrid $\mathrm{P}_{1} \times \mathrm{P}_{3}$ showed maximum heterosis over standard parent $(-11.23 \%)$. With respect to node number of first female flower, parental mean ranged from $12.00\left(\mathrm{P}_{4}\right)$ to $26.67\left(\mathrm{P}_{2}\right)$ node number. Among the crosses it ranged from $9\left(\mathrm{P}_{4} \times \mathrm{P}_{5}\right)$ to $23.67\left(\mathrm{P}_{5} \times \mathrm{P}_{6}\right)$. The extent of heterosis varied from -19.94 to $-30.93 \%$ over better parent, -19.44 to $-25.00 \%$ over standard parent and -9.48 to $-37.93 \%$ over mid parent (Table 2 and 3 ). 
Table.1 Mean performance of parents and $\mathrm{F}_{1} \mathrm{~s}$ for yield traits in sponge gourd

\begin{tabular}{|c|c|c|c|c|c|c|c|c|c|c|}
\hline Genotype & $\begin{array}{l}\text { Days to first } \\
\text { male flower } \\
\text { anthesis }\end{array}$ & $\begin{array}{c}\text { Node } \\
\text { number of } \\
\text { first male } \\
\text { flower }\end{array}$ & $\begin{array}{c}\text { Days to first } \\
\text { female } \\
\text { flower } \\
\text { anthesis }\end{array}$ & $\begin{array}{c}\text { Node } \\
\text { number of } \\
\text { first female } \\
\text { flower }\end{array}$ & $\begin{array}{l}\text { Fruit } \\
\text { length } \\
\text { (cm) }\end{array}$ & $\begin{array}{c}\text { Fruit } \\
\text { weight } \\
\text { (g) }\end{array}$ & $\begin{array}{l}\text { Number } \\
\text { of fruits } \\
\text { per plant }\end{array}$ & $\begin{array}{l}\text { Number } \\
\text { of } \\
\text { primary } \\
\text { branches }\end{array}$ & $\begin{array}{l}\text { Main vine } \\
\text { length (m) }\end{array}$ & $\begin{array}{l}\text { Total } \\
\text { yield } \\
\text { (q./ha.) }\end{array}$ \\
\hline $\mathbf{P}_{1}$ & 67.67 & 8.67 & 77.33 & 19.00 & 19.06 & 164.33 & 13.25 & 7.35 & 8.93 & 66.29 \\
\hline $\mathbf{P}_{2}$ & 67.33 & 10.67 & 73.67 & 26.67 & 18.65 & 136.67 & 12.96 & 7.43 & 9.29 & 52.23 \\
\hline $\mathbf{P}_{3}$ & 52.67 & 4.67 & 54.67 & 15.33 & 21.99 & 255.0 & 12.16 & 7.79 & 9.41 & 95.86 \\
\hline $\mathbf{P}_{4}$ & 51.67 & 4.33 & 59.33 & 12.00 & 21.72 & 194.0 & 13.58 & 7.04 & 8.91 & 84.13 \\
\hline $\mathbf{P}_{5}$ & 65.00 & 9.00 & 70.67 & 14.67 & 19.44 & 125.0 & 14.14 & 8.32 & 10.66 & 58.62 \\
\hline $\mathbf{P}_{6}$ & 59.00 & 6.00 & 61.67 & 13.00 & 18.36 & 121.33 & 19.16 & 6.99 & 10.20 & 74.30 \\
\hline $\mathbf{P}_{1} \times \mathbf{P}_{2}$ & 54.67 & 7.00 & 60.00 & 20.67 & 22.30 & 225.67 & 16.74 & 6.87 & 9.97 & 120.68 \\
\hline $\mathbf{P}_{1} \times \mathbf{P}_{3}$ & 49.33 & 3.67 & 52.67 & 10.67 & 20.74 & 164.0 & 23.04 & 8.53 & 10.00 & 122.78 \\
\hline $\mathbf{P}_{1} \times \mathbf{P}_{4}$ & 54.33 & 4.00 & 58.67 & 16.00 & 19.51 & 159.33 & 20.67 & 8.04 & 9.52 & 107.80 \\
\hline $\mathbf{P}_{1} \times \mathbf{P}_{5}$ & 56.67 & 4.00 & 61.33 & 11.00 & 18.97 & 219.33 & 15.40 & 7.98 & 9.49 & 108.44 \\
\hline $\mathbf{P}_{1} \times \mathbf{P}_{6}$ & 55.33 & 8.67 & 56.00 & 17.33 & 21.65 & 113.33 & 19.34 & 8.04 & 9.46 & 70.99 \\
\hline $\mathbf{P}_{2} \times \mathbf{P}_{3}$ & 54.67 & 5.33 & 58.00 & 15.67 & 27.35 & 255.33 & 18.63 & 9.68 & 11.08 & 152.76 \\
\hline $\mathbf{P}_{2} \times \mathbf{P}_{4}$ & 53.33 & 4.00 & 54.33 & 12.00 & 21.57 & 200.67 & 19.00 & 7.72 & 10.39 & 126.68 \\
\hline $\mathbf{P}_{2} \times \mathbf{P}_{5}$ & 59.33 & 7.00 & 65.33 & 15.33 & 20.15 & 209.33 & 18.36 & 8.37 & 9.45 & 125.11 \\
\hline $\mathbf{P}_{2} \times \mathbf{P}_{6}$ & 69.67 & 7.33 & 77.33 & 18.00 & 20.65 & 150.67 & 15.37 & 8.06 & 9.32 & 73.71 \\
\hline $\mathbf{P}_{3} \times \mathbf{P}_{4}$ & 60.33 & 5.33 & 63.00 & 9.67 & 20.73 & 154.0 & 24.09 & 9.43 & 10.65 & 123.33 \\
\hline $\mathbf{P}_{3} \times \mathbf{P}_{5}$ & 52.33 & 4.33 & 56.33 & 11.00 & 21.76 & 169.0 & 24.82 & 7.36 & 9.91 & 137.90 \\
\hline $\mathbf{P}_{3} \times \mathbf{P}_{6}$ & 51.33 & 5.33 & 55.67 & 14.00 & 24.67 & 236.67 & 18.76 & 6.90 & 9.81 & 142.31 \\
\hline $\mathbf{P}_{4} \times \mathbf{P}_{5}$ & 56.33 & 5.00 & 60.67 & 9.00 & 20.60 & 145.33 & 21.54 & 8.65 & 10.64 & 101.85 \\
\hline $\mathbf{P}_{4} \times \mathbf{P}_{6}$ & 60.33 & 5.67 & 67.67 & 17.00 & 19.83 & 210.0 & 14.30 & 7.95 & 9.35 & 92.64 \\
\hline $\mathbf{P}_{5} \times \mathbf{P}_{6}$ & 59.67 & 12.67 & 66.00 & 23.67 & 20.82 & 199.33 & 22.39 & 8.95 & 9.45 & 137.59 \\
\hline Grand Mean & 57.67 & 6.32 & 62.40 & 15.32 & 20.98 & 181.35 & 17.99 & 7.97 & 9.81 & 103.62 \\
\hline CD at $5 \%$ & 3.09 & 0.73 & 4.32 & 2.14 & 1.57 & 19.74 & 3.51 & 0.74 & 0.79 & 12.55 \\
\hline CD at $1 \%$ & 4.14 & 0.97 & 5.78 & 2.86 & 2.09 & 26.42 & 4.69 & 0.99 & 1.05 & 16.79 \\
\hline $\mathbf{C V}$ & 3.25 & 6.98 & 4.20 & 8.46 & 4.52 & 6.60 & 11.81 & 5.61 & 4.86 & 7.34 \\
\hline
\end{tabular}

$\mathrm{P}_{1}$-PSG-93, $\mathrm{P}_{2}$ - PSG-82, $\mathrm{P}_{3}$ - PSG-07-04, $\mathrm{P}_{4}$ - PSG-115, $\mathrm{P}_{5}$ - PSG-199 and $\mathrm{P}_{6}$ - PSG-161 
Table.2 Range of different yield traits, heterosis percentage and performance of top $\mathrm{F}_{1}$ hybrids

\begin{tabular}{|c|c|c|c|c|c|}
\hline Traits & $\begin{array}{l}\text { Days to first male } \\
\text { flower anthesis }\end{array}$ & $\begin{array}{l}\text { Node number of } \\
\text { first male flower }\end{array}$ & $\begin{array}{l}\text { Days to first } \\
\text { female flower } \\
\text { anthesis }\end{array}$ & $\begin{array}{l}\text { Node number of } \\
\text { first female flower }\end{array}$ & Fruit length (cm) \\
\hline \multicolumn{6}{|l|}{ Range of mean values } \\
\hline Parents & 51.67 to 67.67 & 4.33 to 10.67 & 54.67 to 77.33 & 12.00 to 26.67 & 18.36 to 21.99 \\
\hline $\mathrm{F}_{1}$ & 49.33 to 69.67 & 3.67 to 12.67 & 52.67 to 77.33 & 9.00 to 23.67 & 18.97 to 27.35 \\
\hline \multicolumn{6}{|c|}{ Range of heterosis percentage } \\
\hline $\mathbf{B P}$ & -6.21 to -18.81 & -19.23 to -53.84 & -7.54 to -18.55 & -19.44 to -30.43 & 12.15 to 24.37 \\
\hline $\mathbf{S P}$ & 0 & 0 & -8.42 to -11.23 & -19.44 to -25.00 & 13.56 to 25.93 \\
\hline MP & -8.06 to -19.01 & -12.00 to -54.71 & -6.60 to -20.53 & -9.48 to -37.93 & 6.87 to 34.61 \\
\hline \multicolumn{6}{|c|}{ Number of heterotic crosses over } \\
\hline $\begin{array}{l}\text { BP } \\
\end{array}$ & 5 & 6 & 5 & 5 & 6 \\
\hline SP & 0 & 0 & 2 & 2 & 3 \\
\hline MP & 10 & 10 & 9 & 9 & 7 \\
\hline Three top parents & $\begin{array}{l}\mathrm{P}_{4}(51.67) \\
\mathrm{P}_{3}(52.67) \\
\mathrm{P}_{6}(59.00)\end{array}$ & $\begin{array}{l}\mathrm{P}_{4}(4.33) \\
\mathrm{P}_{3}(4.67) \\
\mathrm{P}_{6}(6.00)\end{array}$ & $\begin{array}{l}\mathrm{P}_{3}(54.67) \\
\mathrm{P}_{4}(59.33) \\
\mathrm{P}_{6}(61.67)\end{array}$ & $\begin{array}{l}\mathrm{P}_{4}(12.00) \\
\mathrm{P}_{6}(13.00) \\
\mathrm{P}_{5}(14.67)\end{array}$ & $\begin{array}{l}\mathrm{P}_{3}(21.99) \\
\mathrm{P}_{4}(21.72) \\
\mathrm{P}_{5}(19.44)\end{array}$ \\
\hline \multicolumn{6}{|c|}{ Three top $\mathrm{F}_{1} \mathrm{~s}$ with heterosis percentage } \\
\hline $\mathbf{B P}$ & $\begin{array}{c}\mathrm{P}_{1} \times \mathrm{P}_{2}(-18.81) \\
\mathrm{P}_{1} \times \mathrm{P}_{5}(-12.82) \\
\mathrm{P}_{2} \times \mathrm{P}_{5}(-8.72)\end{array}$ & $\begin{array}{l}P_{1} \times P_{5}(-53.85) \\
P_{3} \times P_{5}(-51.85) \\
P_{4} \times P_{5}(-44.44)\end{array}$ & $\begin{array}{c}\mathrm{P}_{1} \times \mathrm{P}_{2}(-18.55) \\
\mathrm{P}_{1} \times \mathrm{P}_{5}(-13.21) \\
\mathrm{P}_{1} \times \mathrm{P}_{6}(-9.19)\end{array}$ & $\begin{array}{c}\mathrm{P}_{1} \times \mathrm{P}_{3}(-30.44) \\
\mathrm{P}_{1} \times \mathrm{P}_{5}, \mathrm{P}_{3} \times \mathrm{P}_{5} \text { and } \\
\mathrm{P}_{4} \times \mathrm{P}_{5}(-25.00) \\
\mathrm{P}_{3} \times \mathrm{P}_{4}(-19.44)\end{array}$ & $\begin{array}{l}\mathrm{P}_{2} \times \mathrm{P}_{3}(24.37) \\
\mathrm{P}_{1} \times \mathrm{P}_{6}(17.92) \\
\mathrm{P}_{1} \times \mathrm{P}_{2}(17.00)\end{array}$ \\
\hline $\mathbf{S P}$ & $\begin{array}{l}- \\
-\end{array}$ & $\begin{array}{l}- \\
-\end{array}$ & $\begin{array}{c}\mathrm{P}_{1} \times \mathrm{P}_{3}(-11.23) \\
\mathrm{P}_{2} \times \mathrm{P}_{4}(-8.42) \\
\mathrm{P}_{2} \times \mathrm{P}_{5}(-10.11)\end{array}$ & $\begin{array}{l}\mathrm{P}_{4} \times \mathrm{P}_{5}(-25.00) \\
\mathrm{P}_{3} \times \mathrm{P}_{4}(-19.44) \\
\mathrm{P}_{1} \times \mathrm{P}_{3}(-11.11)\end{array}$ & $\begin{array}{l}\mathrm{P}_{2} \times \mathrm{P}_{3}(25.94) \\
\mathrm{P}_{1} \times \mathrm{P}_{2}(22.65) \\
\mathrm{P}_{3} \times \mathrm{P}_{6}(13.57)\end{array}$ \\
\hline MP & $\begin{array}{l}\mathrm{P}_{1} \times \mathrm{P}_{2}(-19.01) \\
\mathrm{P}_{1} \times \mathrm{P}_{3}(-18.01) \\
\mathrm{P}_{1} \times \mathrm{P}_{5}(14.57)\end{array}$ & $\begin{array}{l}\mathrm{P}_{1} \times \mathrm{P}_{5}(-54.72) \\
\mathrm{P}_{2} \times \mathrm{P}_{4}(-46.67) \\
\mathrm{P}_{1} \times \mathrm{P}_{4}(-38.46)\end{array}$ & $\begin{array}{l}\mathrm{P}_{1} \times \mathrm{P}_{2}(-20.53) \\
\mathrm{P}_{1} \times \mathrm{P}_{3}(-20.20) \\
\mathrm{P}_{1} \times \mathrm{P}_{6}(-19.42)\end{array}$ & $\begin{array}{l}P_{2} \times P_{4}(-37.93) \\
P_{1} \times P_{3}(-37.86) \\
P_{1} \times P_{5}(-34.65)\end{array}$ & $\begin{array}{l}\mathrm{P}_{2} \times \mathrm{P}_{3}(34.61) \\
\mathrm{P}_{3} \times \mathrm{P}_{6}(22.24) \\
\mathrm{P}_{1} \times \mathrm{P}_{2}(18.27)\end{array}$ \\
\hline
\end{tabular}

BP- Better Parent, SP -Standard Parent and MP -Mid parent 
Table.3 Range of different yield traits, heterosis percentage and performance of top $\mathrm{F}_{1}$ hybrids

\begin{tabular}{|c|c|c|c|c|c|}
\hline Traits & Fruit weight (g) & $\begin{array}{l}\text { Number of fruits } \\
\text { per plant }\end{array}$ & $\begin{array}{c}\text { Number of } \\
\text { primary } \\
\text { branches }\end{array}$ & $\begin{array}{l}\text { Main vine length } \\
\text { (m) }\end{array}$ & Total yield (q./ha.) \\
\hline \multicolumn{6}{|l|}{ Range of mean values } \\
\hline Parents & 121.33 to 255.00 & 12.16 to 19.16 & 6.99 to 8.32 & 8.91 to 10.66 & 52.23 to 95.86 \\
\hline $\mathbf{F}_{1}$ & 113.33 to 255.33 & 14.30 to 24.82 & 6.87 to 9.68 & 9.32 to 11.08 & 70.99 to 152.76 \\
\hline \multicolumn{6}{|c|}{ Range of heterosis percentage } \\
\hline $\mathbf{B P}$ & 24.17 to 67.46 & 29.85 to 104.08 & 9.58 to 28.14 & 11.68 to 19.41 & 21.05 to 113.43 \\
\hline $\mathbf{S P}$ & 13.05 to 31.61 & 35.17 to 82.79 & 12.87 to 37.54 & 10.10 to 24.35 & 21.05 to 81.58 \\
\hline MP & 16.79 to 61.84 & 19.35 to 88.77 & 11.77 to 27.27 & 8.73 to 18.46 & 16.94 to 125.74 \\
\hline \multicolumn{6}{|c|}{ Number of heterotic crosses over } \\
\hline $\mathbf{B P}$ & 5 & 9 & 9 & 5 & 12 \\
\hline SP & 4 & 11 & 11 & 8 & 12 \\
\hline $\mathbf{T P}$ & 9 & 12 & 9 & 6 & 12 \\
\hline Three top parents & $\begin{array}{l}\text { P3 }(255.00) \\
\text { P4 (194.00) } \\
\text { P1 (164.33) }\end{array}$ & $\begin{array}{l}\text { P6 (19.16) } \\
\text { P5 (14.14) } \\
\text { P4 (13.58) }\end{array}$ & $\begin{array}{l}\text { P5 }(8.32) \\
\text { P3 }(7.79) \\
\text { P2 }(7.43)\end{array}$ & $\begin{array}{l}\text { P5 }(10.66) \\
\text { P6 }(10.20) \\
\text { P3 }(9.41)\end{array}$ & $\begin{array}{l}\text { P3 }(95.86) \\
\text { P4 (84.13) } \\
\text { P6 (74.30) }\end{array}$ \\
\hline \multicolumn{6}{|c|}{$\begin{array}{l}\text { Three top } \mathrm{F}_{1} \mathrm{~S} \text { with heterosis } \\
\text { percentage }\end{array}$} \\
\hline BP & $\begin{array}{l}\mathrm{P}_{2} \times \mathrm{P}_{5}(67.47) \\
\mathrm{P}_{5} \times \mathrm{P}_{6}(64.29) \\
\mathrm{P}_{1} \times \mathrm{P}_{2}(37.32)\end{array}$ & $\begin{array}{c}\mathrm{P}_{3} \times \mathrm{P}_{5}(104.08) \\
\mathrm{P}_{3} \times \mathrm{P}_{4}(98.03) \\
\mathrm{P}_{1} \times \mathrm{P}_{3}(89.39)\end{array}$ & $\begin{array}{l}\mathrm{P}_{5} \times \mathrm{P}_{6}(28.14) \\
\mathrm{P}_{2} \times \mathrm{P}_{3}(24.36) \\
\mathrm{P}_{4} \times \mathrm{P}_{5}(22.92)\end{array}$ & $\begin{array}{l}\mathrm{P}_{4} \times \mathrm{P}_{5}(19.42) \\
\mathrm{P}_{2} \times \mathrm{P}_{3}(17.71) \\
\mathrm{P}_{2} \times \mathrm{P}_{4}(16.61)\end{array}$ & $\begin{array}{c}\mathrm{P}_{2} \times \mathrm{P}_{5}(113.43) \\
\mathrm{P}_{5} \times \mathrm{P}_{6}(85.18) \\
\mathrm{P}_{1} \times \mathrm{P}_{2}(82.04)\end{array}$ \\
\hline SP & $\begin{array}{l}\mathrm{P}_{2} \times \mathrm{P}_{3}(31.62) \\
\mathrm{P}_{3} \times \mathrm{P}_{6}(21.09) \\
\mathrm{P}_{1} \times \mathrm{P}_{2}(16.32)\end{array}$ & $\begin{array}{l}\mathrm{P}_{3} \times \mathrm{P}_{5}(82.79) \\
\mathrm{P}_{3} \times \mathrm{P}_{4}(77.37) \\
\mathrm{P}_{1} \times \mathrm{P}_{3}(69.64)\end{array}$ & $\begin{array}{l}\mathrm{P}_{2} \times \mathrm{P}_{3}(37.55) \\
\mathrm{P}_{3} \times \mathrm{P}_{4}(33.99) \\
\mathrm{P}_{5} \times \mathrm{P}_{6}(27.18)\end{array}$ & $\begin{array}{l}\mathrm{P}_{2} \times \mathrm{P}_{3}(24.36) \\
\mathrm{P}_{3} \times \mathrm{P}_{4}(19.53) \\
\mathrm{P}_{4} \times \mathrm{P}_{5}(19.42)\end{array}$ & $\begin{array}{l}\mathrm{P}_{2} \times \mathrm{P}_{3}(81.58) \\
\mathrm{P}_{3} \times \mathrm{P}_{6}(69.16) \\
\mathrm{P}_{3} \times \mathrm{P}_{5}(63.91)\end{array}$ \\
\hline MP & $\begin{array}{l}\mathrm{P}_{5} \times \mathrm{P}_{6}(61.84) \\
\mathrm{P}_{2} \times \mathrm{P}_{5}(60.00) \\
\mathrm{P}_{1} \times \mathrm{P}_{5}(51.61)\end{array}$ & $\begin{array}{l}\mathrm{P}_{3} \times \mathrm{P}_{5}(88.77) \\
\mathrm{P}_{3} \times \mathrm{P}_{4}(87.15) \\
\mathrm{P}_{1} \times \mathrm{P}_{3}(81.32)\end{array}$ & $\begin{array}{l}\mathrm{P}_{3} \times \mathrm{P}_{4}(27.28) \\
\mathrm{P}_{2} \times \mathrm{P}_{3}(27.25) \\
\mathrm{P}_{5} \times \mathrm{P}_{6}(16.96)\end{array}$ & $\begin{array}{l}\mathrm{P}_{2} \times \mathrm{P}_{3}(18.46) \\
\mathrm{P}_{3} \times \mathrm{P}_{4}(16.25) \\
\mathrm{P}_{2} \times \mathrm{P}_{4}(14.15)\end{array}$ & $\begin{array}{l}\mathrm{P}_{2} \times \mathrm{P}_{5}(125.74) \\
\mathrm{P}_{5} \times \mathrm{P}_{6}(107.03) \\
\mathrm{P}_{2} \times \mathrm{P}_{3}(106.31)\end{array}$ \\
\hline
\end{tabular}

BP- Better Parent, SP -Standard Parent and MP -Mid parent 
Five hybrids showed negative heterosis over better parent, out of which $\mathrm{P}_{1} \times \mathrm{P}_{3}$ exhibited highest significant negative heterosis over better parent, two hybrids showed negative heterosis over standard parent, out of which $\mathrm{P}_{4} \times \mathrm{P}_{5}$ exhibited highest significant negative heterosis over standard parent $(-25.00 \%)$ and nine hybrids showed negative heterosis over mid parent, out of which $\mathrm{P}_{2} \times \mathrm{P}_{4}$ exhibited highest significant negative heterosis over mid parent $(-37.93 \%)$. Earliness (indicated by negative estimates of heterosis) is a wellrecognized and prime objective of any breeding programme as it helps the grower to reap a high market price earlier. The best crosses $\mathrm{P}_{1} \times \mathrm{P}_{2}, \mathrm{P}_{1} \times \mathrm{P}_{3}, \mathrm{P}_{1} \times \mathrm{P}_{4}, \mathrm{P}_{1} \times \mathrm{P}_{5}, \mathrm{P}_{1} \times$ $\mathrm{P}_{6}, \mathrm{P}_{2} \times \mathrm{P}_{3}, \mathrm{P}_{2} \times \mathrm{P}_{4}, \mathrm{P}_{2} \times \mathrm{P}_{5}, \mathrm{P}_{3} \times \mathrm{P}_{4}, \mathrm{P}_{3} \times \mathrm{P}_{5}$, $\mathrm{P}_{3} \times \mathrm{P}_{6}$ and $\mathrm{P}_{4} \times \mathrm{P}_{5}$ were found to be the most promising for earliness. In accordance with the present findings, Abusaleha and Dutta (1995) found in sponge gourd and Mole et al., (2001); Shaha and Kale (2003) and Rao et al., (2000) also observed earliness in heterotic combinations of ridge gourd.

The estimates of parental mean value for fruit length ranged from $18.38 \mathrm{~cm}\left(\mathrm{P}_{6}\right)$ to $21.99 \mathrm{~cm}$ $\left(\mathrm{P}_{3}\right)$ while for $\mathrm{F}_{1}$ hybrids it varied from $18.97 \mathrm{~cm}\left(\mathrm{P}_{1} \times \mathrm{P}_{5}\right)$ to $27.35 \mathrm{~cm}\left(\mathrm{P}_{2} \times \mathrm{P}_{3}\right)$. The range of heterosis varied from 12.15 to $24.37 \%, 13.56$ to $25.93 \%$ and 6.87 to $34.61 \%$ over better parent, standard parent and mid parent, respectively. Six hybrids showed highly significant heterosis over better parent. The hybrid $\mathrm{P}_{2} \times \mathrm{P}_{3}$ exhibited maximum heterosis $24.37 \%, 25.94 \%$ and $34.61 \%$ over better parent, standard parent and mid parent, respectively. A perusal of average data for fruit weight varied from $121.36 \mathrm{~g}\left(\mathrm{P}_{6}\right)$ to $255.00 \mathrm{~g}\left(\mathrm{P}_{3}\right)$, whereas in crosses it ranged from 113.33g $\left(\mathrm{P}_{1} \times \mathrm{P}_{6}\right)$ to $255.33 \mathrm{~g}\left(\mathrm{P}_{2} \times \mathrm{P}_{3}\right)$. The extent of heterosis varied from 24.84 to $57.46 \%$ over better parent, 13.05 to $31.61 \%$ over standard parent and 16.79 to $61.84 \%$ over mid parent. The highly significant heterosis was noted in 5, 4 and 9 hybrids over better parent, standard parent and mid parent, respectively. The $\mathrm{F}_{1}$ hybrid $\mathrm{P}_{2} \times \mathrm{P}_{5}$ showed maximum heterosis over better parent (67.47\%), $\mathrm{P}_{2} \times \mathrm{P}_{3}$ showed maximum heterosis over standard parent $(31.62 \%)$ and $\mathrm{P}_{5} \times \mathrm{P}_{6}$ showed maximum heterosis over mid parent $(61.84 \%)$. The mean value for number of fruit per plant of parent varied from $12.16\left(\mathrm{P}_{3}\right)$ to $19.16\left(\mathrm{P}_{6}\right)$ and for $\mathrm{F}_{1}$ hybrids it ranged from $14.30\left(\mathrm{P}_{4} \times \mathrm{P}_{6}\right)$ to $24.82\left(\mathrm{P}_{3} \times \mathrm{P}_{5}\right)$. Heterosis ranged from 29.85 to $104.08 \%$ over better parent, 35.17 to $82.79 \%$ over standard parent and 19.35 to $88.77 \%$ over mid parent. Nine crosses were possessing significant heterosis over better parent. The cross $\mathrm{P}_{3} \times \mathrm{P}_{5}$ exhibited maximum heterosis 104.08, 82.79 and $88.77 \%$ over better parent, standard parent and mid parent, respectively. The findings are similar to the result obtained by Ahmed et al., (2006); Singh et al., (2009) and Hedau and Sirohi (2004) reported that yield attributing characters and fruit yield showed positive heterosis, while vine length and flowering showed negative heterosis. The three best performing $F_{1}$ hybrids for yield were DRG-1 $\times$ P.N., DRG-1 $\times$ PRG-7 and DRG-1 $\times$ AAUJ-3 and manifested 93.09, 68.51 and 66.50 percent heterosis over top parent (DRG1), respectively. These hybrids also recorded the maximum fruit weight, fruit length and highest number of fruit per plant in ridge gourd and similar result found by Patel and Desai (2008) in sponge gourd. The result for number of primary branches of parental mean ranged from $6.99\left(\mathrm{P}_{6}\right)$ to $8.32\left(\mathrm{P}_{5}\right)$ and mean of the crosses varied from $6.87\left(\mathrm{P}_{1} \times \mathrm{P}_{2}\right)$ to $9.68\left(\mathrm{P}_{2} \times \mathrm{P}_{3}\right)$. Range of heterosis was estimated from 9.58 to $28.14 \%$ over better parent, 12.87 to $37.54 \%$ over standard parent and 11.77 to $27.27 \%$ over mid parent. Nine crosses were significant superior to the better parent. The hybrid $\mathrm{P}_{5} \times \mathrm{P}_{6}$ showed maximum heterosis $(28.15 \%)$ over better parent, hybrid $\mathrm{P}_{2} \times \mathrm{P}_{3}$ showed maximum heterosis $(37.55 \%)$ over mid parent. The estimates of parental mean value for main vine length ranged from 
8.91m $\left(\mathrm{P}_{4}\right)$ to $10.66 \mathrm{~m}\left(\mathrm{P}_{5}\right)$ while for $\mathrm{F}_{1}$ hybrid it varied from $9.32 \mathrm{~m}\left(\mathrm{P}_{2} \times \mathrm{P}_{6}\right)$ to $11.08 \mathrm{~m}\left(\mathrm{P}_{2}\right.$ $\times \mathrm{P}_{3}$ ). The range of heterosis varied from 11.68 to $19.41 \%, 10.10$ to $24.35 \%$ and 8.73 to $18.46 \%$ over better parent, standard parent and mid parent, respectively. Five hybrids showed highly significant heterosis over better parent. The $\mathrm{F}_{1}$ hybrid $\mathrm{P}_{4} \times \mathrm{P}_{5}$ exhibited maximum heterosis $(19.42 \%)$ over better parent while $\mathrm{F}_{1}$ hybrid $\mathrm{P}_{2} \times \mathrm{P}_{3}$ showed maximum heterosis over standard parent (24.36\%) and mid parent (18.46\%). The mean value for total yield per hectare ranged from $52.23 \mathrm{q} / \mathrm{ha}\left(\mathrm{P}_{2}\right)$ to $95.86 \mathrm{q} / \mathrm{ha}\left(\mathrm{P}_{3}\right)$, whereas for crosses it ranged from $70.99 \mathrm{q} / \mathrm{ha}\left(\mathrm{P}_{1} \times \mathrm{P}_{6}\right)$ to $152.86 \mathrm{q} / \mathrm{ha}\left(\mathrm{P}_{2} \times \mathrm{P}_{3}\right)$. The extent of heterosis for this foremost trait ranged from 21.05 to $113.43 \%$ over better parent, 21.05 to $81.58 \%$ over standard parent and 16.95 to $125.74 \%$ over mid parent. The twelve crosses performed better parent heterosis. The maximum heterosis estimate over better parent was recorded in crosses like $\mathrm{P}_{2} \times \mathrm{P}_{5}$ (113.43\%) followed by $\mathrm{P}_{5} \times \mathrm{P}_{6}(85.18 \%)$ and $\mathrm{P}_{1} \times \mathrm{P}_{2}(82.04 \%)$. Based on the result on standard parent heterosis, it was evident that cross $\mathrm{P}_{2} \times \mathrm{P}_{3}$ exhibited maximum heterosis $(81.58 \%)$ followed by $\mathrm{P}_{3} \times \mathrm{P}_{6}(69.16 \%)$ and $\mathrm{P}_{3} \times \mathrm{P}_{5}(63.91 \%)$. The maximum heterosis estimate over mid parent was recorded in crosses like $\mathrm{P}_{2} \times \mathrm{P}_{5}(125.74 \%)$, followed by $\mathrm{P}_{5}$ $\times \mathrm{P}_{6}(107.03 \%)$ and $\mathrm{P}_{2} \times \mathrm{P}_{3}(106.31 \%)$. Heterotic effect for these fruit characters were also reported by Narasannavar et al., (2014) and Rao et al., (2000) for yield, the $\mathrm{F}_{1} \mathrm{~s}$ LA-46 $\times$ LA-99, LA-12 $\times$ LA-76 and LA-32 $\times$ LA37 proved the best and manifested 51.8 and 125.9, 81.8 and 121.1 and 99.1 and $200.1 \%$ heterosis, respectively, in both kharif and summer seasons over the better parent in ridge gourd and similar result also found in sponge gourd by Patel and Desai (2008). Naliyadhara et al., (2007) revealed that the cross combination CHSG-1 $\times$ JSGL-70 depicted highest heterobeltiosis (69.10\%), whereas the cross combination NSG-28 $\times$
CHSG-1 exhibited highest economic heterosis $(53.56 \%)$ with maximum fruit yield (1.69 $\mathrm{kg} / \mathrm{vine}$ ) in sponge gourd.

The crosses $\mathrm{P}_{2} \times \mathrm{P}_{3}, \mathrm{P}_{3} \times \mathrm{P}_{6}$ and $\mathrm{P}_{3} \times \mathrm{P}_{5}$ were found to be best heterotic combinations as they exhibited significant heterosis percentage for yield per hectare over the standard check parent. The high yielding $\mathrm{F}_{1}$ hybrid $\mathrm{P}_{2} \times \mathrm{P}_{3}$ (PSG-82 $\times$ PSG-07-04) was showed $81.58 \%$ heterosis for yield over standard check may be recommended for commercial exploitation.

\section{References}

Abusaleha and Dutta, O. P. (1995). Studies on heterosis in sponge gourd. Indian Journal of Horticulture, 52 (3): 222-225.

Ahmed, M. A., Reddy, I. P. and Neeraja, G. (2006). Combining ability and heterosis for fruit yield and yield components in ridge gourd (Luffa acutangula). The Journal of Research Angrau, 34 (1): 1520.

Fonseca, S. and Patterson, F. L. (1968). Hybrid vigour in seven parental diallel cross in common wheat (Triticum astivum L.). Crop Science, 8: 85-88.

Hedau, N. K. and Sirohi, P. S. (2004). Heterosis studies in ridge gourd. Indian Journal of Horticulture, 61(3): 236-239.

Kalloo, G. (1993). Loofah-Luffa spp. In: Genetic Improvement of Vegetable Crops. Pergamon Press, pp. 265-266.

Kalloo, G., Banerjee, M.K., Kumar, S. and Parkash, C. (2000). Hybrid Vegetable Technology in India: An overview. In: Emerging Scenario in Vegetable Research and Development. Research Periodicals and Book Publishing House, India, pp. 11-31.

Mole, T. J., Devi, S. N., Rajan, S. and Sadhankumar, P. G. (2001). Heterosis and combining ability in ridge gourd (Luffa acutangula Roxb.). Vegetable Science, 28(2): 165-167. 
Naliyadhara, M. V., Dhaduk, L. K., Barad, A. V., Purohit, V. L. and Vachhani, J. H. (2007). Heterosis for fruit yield and its components in sponge gourd [Luffa cylindrica (Roem.) L.]. National journal of plant improvement, 9 (2): 132-135.

Narasannavar, A. R., Gasti, V. D., Shantappa, T., Mulge, R., Allolli, T. B. and Thammaiah, N. (2014). Heterosis studies in ridge gourd (Luffa acutangula Roxb.). Karnataka Journal of Agricultural Science, 27 (1): 47-51.

Patel, S. R. and Desai, D. T. (2008). Heterosis and combining ability studies in sponge gourd [Luffa cylindrica (Roem.) L.]. Vegetable Science, 35(2):199-200.
Rao, B. N., Rao, P. V. and Reddy, B. M. (2000). Heterosis in ridge gourd (Luffa acutangula (L.) Roxb.). Haryana Journal of Horticultural Science, 29(1/2): 96-98.

Shaha, S. R. and Kale, P. N. (2003). Heterosis studies in ridge gourd. Journal of Maharashtra Agricultural Universities, 28(3): 334-336.

Singh, P. K., Choudhary, B. R., Bhardwaj, D. R. and Singh, R. (2009). Heterosis response in sponge gourd (Luffa cylindrica Roem). Indian Journal of Arid Horticulture, 4 (2): 118-119.

Yawalkar, K.S. (2004). Cucurbitaceous or vine crops. Vegetable Crops of India (V Ed.), pp. 152-155.

\section{How to cite this article:}

Vijay Bahadur Singh Chauhan, Dinesh Kumar Singh and Harshawardhan Choudhary. 2018. Studies on Heterosis for Yield and its Contributing Traits in Sponge Gourd (Luffa cylindrica Roem.). Int.J.Curr.Microbiol.App.Sci. 7(12): 223-230. doi: https://doi.org/10.20546/ijcmas.2018.712.028 